\title{
Fixed Point Theorem in 2 - Metric spaces of implicit Relations
}

\author{
Rajesh Shrivastava ${ }^{1}$, Neha Jain ${ }^{2}$, K. Qureshi ${ }^{3}$ \\ ${ }^{1}$ Deptt .of Mathematics, Govt. Science and comm. College Banazeer Bhopal( M.P) India \\ ${ }^{2}$ Research Scholar, Govt. Science and comm. College Banazeer Bhopal( M.P) India \\ ${ }^{3}$ Additional Director, Higher Education Deptt. Govt. of M.P., Bhopal (M.P) India
}

\section{Email address:}

capricone.neha@gmail.com(N. Jain)

\section{To cite this article:}

Rajesh Shrivastava, Neha Jain, K. Qureshi. Fixed Point Theorem in 2 - Metric Spaces of Implicit Relations. American Journal of Applied Mathematics. Vol. 1, No. 4, 2013, pp. 44-48. doi: 10.11648/j.ajam.20130104.12

Abstract: In this paper present on Fixed point Theorem in 2-metric spaces .A concept which has been in focus recent times. The result is supported with an example.

Keywords: Metric Spaces, Fixed Points, Complete Metric Spaces, Cauchy Sequence

\section{Introduction}

Theorem: -

let $(\mathrm{X}, \mathrm{d})$ and $(\mathrm{Y}, \rho)$ be a complete metric space if $\mathrm{T}: \mathrm{X} \rightarrow \mathrm{Y}$ and $\mathrm{S}: \mathrm{Y} \rightarrow \mathrm{X}$ satisfying the Conditions :

\section{1. [Fisher [1]]}

$\mathrm{d}($ Sy, STx $) \leq \mathrm{c} \max \{\mathrm{d}(\mathrm{x}, \mathrm{Sy}), \mathrm{d}(\mathrm{x}, \mathrm{STx}), \rho(\mathrm{y}, \mathrm{Tx})\}$

$\rho(\mathrm{Tx}, \mathrm{TSy}) \leq \mathrm{c} \max \{\rho(\mathrm{y}, \mathrm{Tx}), \rho(\mathrm{y}, \mathrm{TSy}), \mathrm{d}(\mathrm{x}$, Sy $)\}$

for all $\mathrm{x}$ in $\mathrm{X}$ and $\mathrm{y}$ in $\mathrm{Y}$, where $0 \leq \mathrm{c}<1$,

\section{2. [Popa [3]]}

$$
\begin{array}{r}
d^{2}(\text { Sy }, \text { STx }) \leq c_{1} \max \{\rho(y, T x) d(x, S y), \rho(y, T S y) \\
d(x, S T x), d(x, S y) d(x, S T x)\} \\
\rho^{2}(T x, T S y) \leq c_{2} \max \{d(x, S y) \rho(y, T x), d(x, S T x) \\
\rho(y, T S y), \rho(y, T x) \rho(y, T S y)\}
\end{array}
$$

for all $\mathrm{x}$ in $\mathrm{X}$ and $\mathrm{y}$ in $\mathrm{Y}$, where $0 \leq \mathrm{c}_{1}, \mathrm{c}_{2}<1$.

\section{3. [(Nešić[2]]}

$\mathrm{M}_{1}(\mathrm{x}, \mathrm{y})=\left\{\mathrm{d}^{\mathrm{p}}(\mathrm{x}, \mathrm{Sy}), \mathrm{d}^{\mathrm{p}}(\mathrm{x}, \mathrm{ST} \mathrm{x}), \rho^{\mathrm{p}}(\mathrm{y}, \mathrm{Tx})\right\}$

$\mathrm{M}_{2}(\mathrm{x}, \mathrm{y})=\left\{\rho^{\mathrm{p}}(\mathrm{y}, \mathrm{Tx}), \rho^{\mathrm{p}}(\mathrm{y}, \mathrm{TSy}), \mathrm{d}^{\mathrm{p}}(\mathrm{x}\right.$, Sy $\left.)\right\}$

for all $\mathrm{x}$ in $\mathrm{X}, \mathrm{y}$ in $\mathrm{Y}$ and $\mathrm{p}=1,2,3, \ldots \ldots$.

let $\mathrm{R}^{+}$be the set of nonnegative real numbers, and let

$\mathrm{F}_{\mathrm{i}}: \mathrm{R}^{+} \rightarrow \mathrm{R}^{+}$be a mapping such that

$\mathrm{F}_{\mathrm{i}}(0)=0$ and $\mathrm{F}_{\mathrm{i}}$ is continuous at 0 for $\mathrm{i}=1$,2. If $\mathrm{T}$ and

$\mathrm{S}$ satisfying the inequalities
$\mathrm{d}^{\mathrm{p}}(\mathrm{Sy}, \mathrm{STx}) \leq \mathrm{c}_{1} \max \mathrm{M}_{1}(\mathrm{x}, \mathrm{y})+\mathrm{F}_{1}\left(\min \mathrm{M}_{1}(\mathrm{x}, \mathrm{y})\right)$

$\rho^{\mathrm{p}}(\mathrm{Tx}, \mathrm{TSy}) \leq \mathrm{c}_{2} \max \mathrm{M}_{2}(\mathrm{x}, \mathrm{y})+\mathrm{F}_{2}\left(\min \mathrm{M}_{2}(\mathrm{x}, \mathrm{y})\right)$

for all $\mathrm{x}$ in $\mathrm{X}$ and $\mathrm{y}$ in $\mathrm{Y}$, where $0 \leq \mathrm{c}_{1}, \mathrm{c}_{2}<1$.

Then ST has a unique fixed point $\mathrm{z}$ in $\mathrm{X}$ and TS has a unique fixed point $\mathrm{w}$ inY .

Futher, $\mathrm{Tz}=\mathrm{w}$ and $\mathrm{Sw}=\mathrm{z}$.

\section{Main Results}

The theorem that we are attempting to prove generalizes that Fisher [1] ,Nešić[2] , Popa [3]theorem using an implicit relations . Let $\Phi_{3}{ }^{(\mathrm{m})}$ be the set of continuous functions with 3 variables

$\varphi:[0, \infty)^{3} \rightarrow[0, \infty)$ satisfying the properties:

a. $\varphi$ is non decreasing in $\mathrm{t}_{1}, \mathrm{t}_{2}, \mathrm{t}_{3}$.

b. $\varphi(t, t, t) \leq t^{m} \quad m \in N$

Some examples of such functions are as follows:

Example 2.1 $\psi\left(\mathrm{t}_{1}, \mathrm{t}_{2}, \mathrm{t}_{3}\right)=\min \left\{t_{1}^{p}, t_{2}^{p}, t_{3}^{p}\right\}$

Example 2.2 $\quad \psi\left(\mathrm{t}_{1}, \mathrm{t}_{2}, \mathrm{t}_{3}\right)=\min \left\{\mathrm{t}_{1} \mathrm{t}_{2}, \mathrm{t}_{1} \mathrm{t}_{3}, \mathrm{t}_{2} \mathrm{t}_{3}\right\}$

Example 2.3 $\quad \psi\left(\mathrm{t}_{1}, \mathrm{t}_{2}, \mathrm{t}_{3}\right)=\min \left\{\mathrm{t}_{1}, \mathrm{t}_{2}, \mathrm{t}_{3}\right\}$

Example $2.4 \varphi\left(\mathrm{t}_{1}, \mathrm{t}_{2}, \mathrm{t}_{3}\right)=\max \left\{t_{1}^{p}, t_{2}^{p}, t_{3}^{p}\right\}$,

with $\mathrm{m}=\mathrm{p}$

Example2.5 $\varphi\left(\mathrm{t}_{1}, \mathrm{t}_{2}, \mathrm{t}_{3}\right)=\max \left\{\mathrm{t}_{1}, \mathrm{t}_{2}, \mathrm{t}_{3}\right\}$, with $\mathrm{m}=1$ etc.

Let $F$ be a set of continuous functions $F:[0, \infty) \rightarrow[0, \infty)$ with $\mathrm{F}(0)=0$ (for example $\mathrm{F}(\mathrm{t})=\mathrm{t}^{\mathrm{q}}, \mathrm{q}>0$ ). 


\section{Main theorem}

\section{Theorem 3.1}

Let $(\mathrm{X}, \mathrm{d}),(\mathrm{Y}, \rho)$ be two complete metric spaces and $\mathrm{T}: \mathrm{X} \rightarrow Y, \mathrm{~S}: \mathrm{Y} \rightarrow \mathrm{X}$ two mappings . Let $\varnothing \in \Phi_{3}^{(m)}$, $\psi_{\mathrm{i}} \in \Psi_{3}, \mathrm{~F}_{\mathrm{i}} \in f$ for $i=1,2$. if for some $k \in[0,1)$, the following inequalities are satisfied:

$$
\begin{aligned}
& \mathrm{d}^{\mathrm{m}}(\mathrm{Sy}, \mathrm{STx}) \leq \mathrm{k} \varphi_{1}(\mathrm{~d}(\mathrm{x}, \mathrm{STx}), \rho(y, T x))+ \\
& \mathrm{F}_{1}\left(\psi_{1}(\mathrm{~d}(\mathrm{x}, \mathrm{STx}), \rho(y, T x))\right) \\
& \rho^{\mathrm{m}}(\mathrm{Tx}, \mathrm{TSy}) \leq \mathrm{k} \varphi_{2}(\rho(y, T S y), d(x, S y))+ \\
& \mathrm{F}_{2}\left(\psi_{2}(\rho(y, T S y), d(x, S y))\right)
\end{aligned}
$$

for all $\mathrm{x} \in \mathrm{X}, \mathrm{y} \in \mathrm{Y}$ and some $\mathrm{m}=1,2, \ldots \ldots$.,

then ST has a unique fixed point $\alpha \in X$ and TS has a unique fixed point $\beta \in Y$. Further $T_{\alpha} \in \beta$ and $S_{\beta} \in \alpha$.

Proof :- let $\mathrm{x}_{0} \in \mathrm{X}$ be an arbitrary point .we define the sequences $\left(\mathrm{x}_{\mathrm{n}}\right)$ and $\left(\mathrm{y}_{\mathrm{n}}\right)$ in $\mathrm{X}$ and $\mathrm{Y}$ respectively as follows :

$$
\mathrm{x}_{\mathrm{n}}=(\mathrm{ST})^{\mathrm{n}} \mathrm{x}_{0}, \mathrm{y}_{\mathrm{n}}=\mathrm{Tx}_{\mathrm{n}-1}, \mathrm{n}=1,2,3 \ldots \ldots,
$$

Let us proof that the sequences $\left(\mathrm{x}_{\mathrm{n}}\right)$ and $\left(\mathrm{y}_{\mathrm{n}}\right)$ are Cauchy sequences. We assume that $\mathrm{x}_{\mathrm{n}} \neq \mathrm{x}_{\mathrm{n}+1}$ and $\mathrm{y}_{\mathrm{n}} \neq \mathrm{y}_{\mathrm{n}+1} \forall \mathrm{n} \in \mathrm{N}$, because otherwise if $x_{n}=x_{n+1}$ and $y_{n}=y_{n+1}$ for some $n$, we could put $\alpha=x_{n}$ and $\beta=y_{n}$.

denote

$\mathrm{d}_{\mathrm{n}}=\mathrm{d}\left(\mathrm{x}_{\mathrm{n}}, \mathrm{x}_{\mathrm{n}+1}\right), \rho_{\mathrm{n}}=\rho\left(\mathrm{y}_{\mathrm{n}}, \mathrm{y}_{\mathrm{n}+1}\right), \mathrm{n}=1,2$

By the inequality (2) for $\mathrm{x}=\mathrm{x}_{\mathrm{n}-1}$ and $\mathrm{y}=\mathrm{y}_{\mathrm{n}}$ we get :

$$
\begin{aligned}
\rho^{\mathrm{m}}\left(\mathrm{y}_{\mathrm{n}}, \mathrm{y}_{\mathrm{n}+1}\right)= & \rho^{\mathrm{m}}\left(\mathrm{Tx}_{\mathrm{n}-1}, \mathrm{TSy}_{\mathrm{n}}\right) \\
\leq & k \varphi_{2}\left(\rho\left(\mathrm{y}_{\mathrm{n}}, \mathrm{TSy}_{\mathrm{n}}\right), \mathrm{d}\left(\mathrm{x}_{\mathrm{n}-1}, \mathrm{Sy}_{\mathrm{n}}\right)\right) \\
& +\mathrm{F}_{2}\left(\psi_{2}\left(\rho\left(\mathrm{y}_{\mathrm{n}}, \mathrm{TSy}_{\mathrm{n}}\right), \mathrm{d}\left(\mathrm{x}_{\mathrm{n}-1}, \mathrm{Sy}_{\mathrm{n}}\right)\right)\right) \\
\rho^{\mathrm{m}}\left(\mathrm{y}_{\mathrm{n}}, \mathrm{y}_{\mathrm{n}+1}\right) \leq & k \varphi_{2}\left(\rho\left(\mathrm{y}_{\mathrm{n}}, \mathrm{y}_{\mathrm{n}+1}\right), \mathrm{d}\left(\mathrm{x}_{\mathrm{n}-1}, \mathrm{x}_{\mathrm{n}}\right)\right) \\
& +\mathrm{F}_{2}\left(\psi_{2}\left(\rho\left(\mathrm{y}_{\mathrm{n}}, \mathrm{y}_{\mathrm{n}+1}\right), \mathrm{d}\left(\mathrm{x}_{\mathrm{n}-1}, \mathrm{x}_{\mathrm{n}}\right)\right)\right)
\end{aligned}
$$

or

$$
\begin{aligned}
& \rho_{n}^{m} \leq k \varphi_{2}\left(\rho_{\mathrm{n}}, \mathrm{d}_{\mathrm{n}-1}\right)+\mathrm{F}_{2}\left(\psi_{2}\left(\rho_{\mathrm{n}}, \mathrm{d}_{\mathrm{n}-1}\right)\right) \\
& =k \varphi_{2}\left(\rho_{\mathrm{n}}, \mathrm{d}_{\mathrm{n}-1}\right)
\end{aligned}
$$

For the coordinates of the point $\left(\rho_{\mathrm{n}}, \mathrm{d}_{\mathrm{n}-1}\right)$ we have : $\rho_{\mathrm{n}} \leq \mathrm{d}_{\mathrm{n}-1}, \forall n \in N$, because ; in case that

$\rho_{\mathrm{n}}>\mathrm{d}_{\mathrm{n}-1}$ for some $\mathrm{n}$, if we replace the coordinates with $\rho_{\mathrm{n}}$ and apply the property (a) and (b) of $\varphi_{2}$ we get

$$
\rho_{n}^{m} \leq k \varphi_{2}\left(\rho_{\mathrm{n}}, \rho_{\mathrm{n}}\right) \leq \mathrm{k} \rho_{n}^{m}
$$

This is impossible since $0 \leq \mathrm{k}<1$.

Replacing on the right hand side of (3), the coordinates with $\rho_{\mathrm{n}-1}$ and applying properties (a) and (b) of $\varphi_{2}$ we get :

$$
\begin{gathered}
\rho_{n}^{m} \leq k \varphi_{2}\left(\mathrm{~d}_{\mathrm{n}-1}, \mathrm{~d}_{\mathrm{n}-1}\right) \leq \mathrm{k} d_{n-1}^{m} \\
\text { Thus } \quad \rho_{\mathrm{n}} \leq \sqrt[m]{\mathrm{k}} \mathrm{d}_{\mathrm{n}-1}
\end{gathered}
$$

By the inequality (1) for $\mathrm{x}=\mathrm{x}_{\mathrm{n}}$ and $\mathrm{y}=\mathrm{y}_{\mathrm{n}}$ we get $d^{m}\left(x_{n}, x_{n+1}\right)=d^{m}\left(\operatorname{Sy}_{n}, \operatorname{STx}_{n}\right)$

$$
\begin{aligned}
\leq & \mathrm{k} \varphi_{1}(\mathrm{~d}(\mathrm{x}, \mathrm{STx}), \rho(y, T x)) \\
& +\mathrm{F}\left(\psi_{1}(\mathrm{~d}(\mathrm{x}, \mathrm{STx}), \rho(\mathrm{y}, \mathrm{Tx}))\right) \\
\leq & \mathrm{k} \varphi_{1}\left[\mathrm{~d}\left(\mathrm{x}_{\mathrm{n}}, \mathrm{x}_{\mathrm{n}+1}\right), \rho\left(\mathrm{y}_{\mathrm{n}}, \mathrm{y}_{\mathrm{n}+1}\right)\right) \\
& +\mathrm{F}_{1}\left(\psi_{1}\left(\mathrm{~d}\left(\mathrm{x}_{\mathrm{n}}, \mathrm{x}_{\mathrm{n}+1}\right), \rho\left(\mathrm{y}_{\mathrm{n}}, \mathrm{y}_{\mathrm{n}+1}\right)\right)\right)
\end{aligned}
$$

Or

$d_{n}^{m} \leq \mathrm{k} \varphi_{1}\left(\mathrm{~d}_{\mathrm{n}}, \rho_{\mathrm{n}}\right)+\mathrm{F}_{1}\left(\psi_{1}\left(\mathrm{~d}_{\mathrm{n}}, \rho_{\mathrm{n}}\right)\right)$

$$
=\mathrm{k} \varphi_{1}\left(\mathrm{~d}_{\mathrm{n}}, \rho_{\mathrm{n}}\right)
$$

In similar, we get :

$$
d_{n}^{m} \leq \mathrm{k} \varphi_{1}\left(\rho_{\mathrm{n}}, \rho_{\mathrm{n}}\right) \leq \mathrm{k} \rho_{n}^{m}
$$

Thus we will obtain :

$\mathrm{d}_{\mathrm{n}} \leq \mathrm{m} \sqrt{\mathrm{k}} \rho_{\mathrm{n}}$

By this inequality and (4) we get :

$$
\begin{aligned}
& \mathrm{d}_{\mathrm{n}} \leq \mathrm{m} \sqrt{\mathrm{k}}\left({ }^{\mathrm{m}} \sqrt{\mathrm{k} \mathrm{d}_{\mathrm{n}-1}}\right) \\
& \leq \mathrm{m} \sqrt{\mathrm{k}} \mathrm{d}_{\mathrm{n}-1}
\end{aligned}
$$

by the inequalities (4) and (5), using the mathematical induction, we obtain :

$$
\begin{aligned}
& \mathrm{d}\left(\mathrm{x}_{\mathrm{n}}, \mathrm{x}_{\mathrm{n}+1}\right)=\mathrm{d}_{\mathrm{n}} \leq \mathrm{q}^{\mathrm{n}-1} \mathrm{~d}_{\mathrm{i}} \\
& \rho\left(\mathrm{y}_{\mathrm{n}}, \mathrm{y}_{\mathrm{n}+1}\right)=\rho_{\mathrm{n}} \leq \mathrm{q}^{\mathrm{n}-1} \mathrm{~d}_{1} \text { Where } \mathrm{q}=\mathrm{m}_{\mathrm{k}}<1
\end{aligned}
$$

Thus the sequences $\left(\mathrm{x}_{\mathrm{n}}\right)$ and $\left(\mathrm{y}_{\mathrm{n}}\right)$ are Cauchy sequences , since the Metric space Let $(\mathrm{X}, \mathrm{d}),(\mathrm{Y} \rho)$ complete metric spaces we have :

$$
\begin{aligned}
& \lim _{n \rightarrow \infty} x_{n}=\alpha \in \mathrm{X}, \lim _{n \rightarrow \infty} y_{n}=\beta \in \mathrm{Y} \\
& \text { by (1) for } y=\beta \text { and } x=x_{n} \text { we get : } \\
& \mathrm{d}^{\mathrm{m}}\left(\mathrm{S} \beta, \mathrm{x}_{\mathrm{n}+1}\right) \leq \mathrm{k} \varphi_{1}\left(\mathrm{~d}\left(\mathrm{x}_{\mathrm{n}}, \mathrm{x}_{\mathrm{n}+1}\right), \rho\left(\beta, \mathrm{y}_{\mathrm{n}+1}\right)\right) \\
& +\mathrm{F}_{1}\left(\psi_{1}\left(\mathrm{~d}\left(\mathrm{x}_{\mathrm{n}}, \mathrm{x}_{\mathrm{n}+1}\right), \rho\left(\beta, \mathrm{y}_{\mathrm{n}+1}\right)\right)\right)
\end{aligned}
$$

Or

$\mathrm{d}^{\mathrm{m}}(\mathrm{S} \beta, \alpha)=\Leftrightarrow \mathrm{S} \beta=\alpha$

by (2) for $\mathrm{x}=\mathrm{x}_{\mathrm{n}}$ and $\mathrm{y}=\beta$ we get :

$$
\begin{aligned}
\rho^{\mathrm{m}}\left(\mathrm{y}_{\mathrm{n}+1}, \mathrm{TS} \beta\right) \leq & k \varphi_{2}\left(\rho(\beta, \mathrm{TS} \beta), \mathrm{d}\left(\mathrm{x}_{\mathrm{n}}, \mathrm{S} \beta\right)\right) \\
& +\mathrm{F}_{2}\left(\psi_{2}\left(\rho(\beta, \mathrm{TS} \beta), \mathrm{d}\left(\mathrm{x}_{\mathrm{n}}, \mathrm{S} \beta\right)\right)\right)
\end{aligned}
$$

letting $\mathrm{n} \rightarrow \infty$, and using(6) we get :

$\rho^{\mathrm{m}}(\beta, \mathrm{TS} \beta) \leq k \varphi_{2}(\rho(\beta, \mathrm{TS} \beta), 0)+\mathrm{F}(0)$

or

$\rho^{\mathrm{m}}(\beta, \mathrm{TS} \beta) \leq k \rho^{\mathrm{m}}(\beta, \mathrm{TS} \beta) \Leftrightarrow \mathrm{TS} \beta=\beta$

by (6) and (7) it follows :

$\mathrm{TS} \beta=\mathrm{T} \alpha=\beta$

$\mathrm{ST} \alpha=\mathrm{S} \beta=\alpha$

Thus we proved that the points $\alpha, \beta$ are fixed points of ST and TS respectively . 


\section{UNIQUENESS}

suppose that ST has a second distinct Fixed point $\alpha^{\prime}$ in X.

By (1) for $\mathrm{x}=\alpha^{\prime}$ and $\mathrm{y}=\mathrm{T} \alpha$ we get :

$$
\begin{aligned}
\mathrm{d}^{\mathrm{m}}\left(\mathrm{ST} \alpha, \mathrm{ST} \alpha^{\prime}\right) \leq \mathrm{k} \varphi_{1}\left(\mathrm{~d}\left(\alpha^{\prime}, \mathrm{ST} \alpha^{\prime}\right), \rho\left(\mathrm{T} \alpha, \mathrm{T} \alpha^{\prime}\right)\right) \\
+\mathrm{F}_{1}\left(\psi_{1}\left(0, \rho\left(\mathrm{T} \alpha, \mathrm{T} \alpha^{\prime}\right)\right)\right)
\end{aligned}
$$

Or

$$
\begin{aligned}
\mathrm{d}^{\mathrm{m}}\left(\alpha, \alpha^{\prime}\right) & \leq \mathrm{k} \varphi_{1} d\left(0, \rho\left(\mathrm{T} \alpha, \mathrm{T} \alpha^{\prime}\right)\right)+\mathrm{F}_{1}(0) \\
& \leq \mathrm{k} \rho^{\mathrm{m}}\left(\mathrm{T} \alpha, \mathrm{T} \alpha^{\prime}\right)
\end{aligned}
$$

By (2) for $\mathrm{x}=\alpha^{\prime}$ and $\mathrm{y}=\mathrm{T} \alpha$ we obtain :

$$
\begin{aligned}
\rho^{\mathrm{m}}\left(\mathrm{T} \alpha^{\prime}, \mathrm{T} \alpha\right) \leq & k \varphi_{2}\left(\rho(\mathrm{T} \alpha, \mathrm{T} \alpha), \mathrm{d}\left(\alpha^{\prime}, \alpha\right)\right) \\
& +\mathrm{F}_{2}\left(\psi_{2}\left(0, \mathrm{~d}\left(\alpha, \alpha^{\prime}\right)\right)\right.
\end{aligned}
$$

or

$$
\begin{aligned}
\rho^{\mathrm{m}}\left(\mathrm{T} \alpha^{\prime}, \mathrm{T} \alpha\right) & \leq k \varphi_{2}\left(\rho\left(0, \mathrm{~d}\left(\alpha^{\prime}, \alpha\right)\right)\right. \\
& \leq \operatorname{kd}^{\mathrm{m}}\left(\alpha^{\prime}, \alpha\right)
\end{aligned}
$$

By (8) and (9) we get :

$\mathrm{d}^{\mathrm{m}}\left(\alpha, \alpha^{\prime}\right) \leq \mathrm{k}^{2} \mathrm{~d}^{\mathrm{m}}\left(\alpha, \alpha^{\prime}\right)$

it follows

$\mathrm{d}\left(\alpha, \alpha^{\prime}\right)=0$

Thus we have again $\alpha=\alpha^{\prime}$. in same way, it is proved the inequality of $\beta$

\section{Hence Proved}

\section{Theorem 3.2}

Let $(\mathrm{X}, \mathrm{d}),(\mathrm{Y}, \rho)$ be two complete metric spaces and $\mathrm{T}: \mathrm{X} \rightarrow Y, \mathrm{~S}: \mathrm{Y} \rightarrow \mathrm{X}$ two mappings. Let $\emptyset \in \Phi_{3}^{(\mathrm{m})}$, $\psi_{\mathrm{i}} \in \Psi_{3}, \mathrm{~F}_{\mathrm{i}} \in f$ for $i=1,2$. if for some $k \in[0,1)$, the following inequalities are satisfied :

$d^{\mathrm{m}}(\mathrm{Sy}, \mathrm{STx}) \leq \mathrm{k} \varphi_{1}(\mathrm{~d}(\mathrm{x}, \mathrm{Sy}), \mathrm{d}(\mathrm{x}, \mathrm{STx}), \rho(y, T x)$, $\left.\frac{\mathrm{d}(\mathrm{x}, \mathrm{Sy}) \mathrm{d}(\mathrm{x}, \mathrm{ST} \mathbf{T})\{1+\rho(\boldsymbol{y}, \mathbf{T} x)\}}{1+\mathrm{d}(\mathrm{x}, \mathrm{Sy})}\right)$

$+F_{1}\left(\psi_{1}(d(x, S y), d(x, S T x)\right.$, $\left.\left.\rho(y, T x), \frac{d(x, S y) d(x, S T x)\{1+\rho(y, T x)\}}{1+d(x, S y)}\right)\right)$

$$
\begin{aligned}
\rho^{\mathrm{m}}(\mathrm{Tx}, \mathrm{TSy}) \leq & \mathbf{k} \varphi_{2}(\rho(y, T x), \rho(y, T S y), d(x, S y), \\
& \left.\left.\frac{\rho(y, T x) d(x, S y)\{1+\rho(y, T S y)\}}{1+\rho(y, T x)}\right)\right) \\
& +\mathrm{F}_{2}\left(\Psi_{2}(\rho(y, T x), \rho(y, T S y), d(x, S y),\right. \\
& \left.\left.\frac{\rho(y, T x) d(x, S y)\{1+\rho(y, T S y)\}}{1+\rho(y, T x)}\right)\right)
\end{aligned}
$$

for all $\mathrm{x} \in \mathrm{X}, \mathrm{y} \in \mathrm{Y}$ and some $\mathrm{m}=1,2, \ldots \ldots$.,then ST has a unique fixed point $\alpha \in X$ and

TS has a unique fixed point $\beta \in \mathrm{Y}$. Futher $\mathrm{T}_{\alpha} \in \beta$ and
$\mathrm{S}_{\beta} \in \alpha$.

Proof :- let $\mathrm{x}_{0} \in \mathrm{X}$ be an arbitrary point . we define the sequences $\left(\mathrm{x}_{\mathrm{n}}\right)$ and $\left(\mathrm{y}_{\mathrm{n}}\right)$ in $\mathrm{X}$ and $\mathrm{Y}$ respectively as follows :

$\mathrm{x}_{\mathrm{n}}=(\mathrm{ST})^{\mathrm{n}} \mathrm{x}_{0}, \mathrm{y}_{\mathrm{n}}=\mathrm{Tx}_{\mathrm{n}-1}, \mathrm{n}=1,2,3 \ldots \ldots$,

Let us proof that the sequences $\left(\mathrm{x}_{\mathrm{n}}\right)$ and $\left(\mathrm{y}_{\mathrm{n}}\right)$ are Cauchy sequences.

We assume that $x_{n} \neq x_{n+1}$ and $y_{n} \neq y_{n+1} \forall n \in N$,

because otherwise if $x_{n}=x_{n+1}$ and $y_{n}=y_{n+1}$ for some $n$, we could put $\alpha=x_{n}$ and $\beta=y_{n}$.

denote $\mathrm{d}_{\mathrm{n}}=\mathrm{d}\left(\mathrm{x}_{\mathrm{n}}, \mathrm{x}_{\mathrm{n}+1}\right), \rho_{\mathrm{n}}=\rho\left(\mathrm{y}_{\mathrm{n}}, \mathrm{y}_{\mathrm{n}+1}\right)$,

$\mathrm{n}=1,2$

By the inequality (2) for $\mathrm{x}=\mathrm{x}_{\mathrm{n}-1}$ and $\mathrm{y}=\mathrm{y}_{\mathrm{n}}$ we get :

$$
\begin{aligned}
& \rho^{\mathrm{m}}\left(\mathrm{y}_{\mathrm{n}}, \mathrm{y}_{\mathrm{n}+1}\right)=\rho^{\mathrm{m}}\left(\mathrm{Tx}_{\mathrm{n}-1}, \mathrm{TSy}_{\mathrm{n}}\right) \\
& \leq k \varphi_{2}\left(\rho\left(\mathrm{y}_{\mathrm{n}}, \mathrm{Tx}_{\mathrm{n}-1}\right), \rho\left(\mathrm{y}_{\mathrm{n}}, \mathrm{TSy}_{\mathrm{n}}\right), \mathrm{d}\left(\mathrm{x}_{\mathrm{n}-1}, \mathrm{Sy}_{\mathrm{n}}\right)\right. \text {, } \\
& \frac{\rho\left(\mathrm{y}_{\mathrm{n}}, \mathrm{Tx}_{\mathrm{n}-1}\right) \mathrm{d}\left(\mathrm{x}_{\mathrm{n}-1}, \mathrm{Sy}_{\mathrm{n}}\right)\left\{1+\rho\left(y, T S y_{n}\right)\right\}}{1+\rho\left(\mathrm{y}_{\mathrm{n}}, \mathrm{Tx}_{\mathrm{n}-1}\right)} \\
& +\mathrm{F}_{2}\left(\psi _ { 2 } \left(\rho\left(\mathrm{y}_{\mathrm{n}}, \mathrm{Tx}_{\mathrm{n}-1}\right), \rho\left(\mathrm{y}_{\mathrm{n}}, \mathrm{TSy}_{\mathrm{n}}\right), \mathrm{d}\left(\mathrm{x}_{\mathrm{n}-1}, \mathrm{Sy}_{\mathrm{n}}\right)\right.\right. \text {, } \\
& \left.\left.\frac{\rho\left(\mathrm{y}_{\mathrm{n}}, \mathrm{Tx}_{\mathrm{n}-1}\right) \mathrm{d}\left(\mathrm{x}_{\mathrm{n}-1}, \mathrm{Sy}_{\mathrm{n}}\right)\left\{1+\rho\left(y, T S y_{n}\right)\right\}}{1+\rho\left(\mathrm{y}_{\mathrm{n}}, \mathrm{Tx}_{\mathrm{n}-1}\right)}\right)\right) \\
& \rho^{m}\left(y_{n}, y_{n+1}\right) \leq k \varphi_{2}\left(\rho\left(y_{n}, y_{n}\right), \rho\left(y_{n}, y_{n+1}\right), d\left(x_{n-1}, x_{n}\right),\right. \\
& \left.\frac{\rho\left(\mathrm{y}_{\mathrm{n}}, \mathrm{y}_{\mathrm{n}}\right) \mathrm{d}\left(\mathrm{x}_{\mathrm{n}-1}, \mathrm{x}_{\mathrm{n}}\right)\left\{1+\rho\left(\mathrm{y}_{\mathrm{n}}, \mathrm{y}_{\mathrm{n}+1}\right)\right\}}{1+\rho\left(\mathrm{y}_{\mathrm{n}}, \mathrm{y}_{\mathrm{n}}\right)}\right) \\
& +\mathrm{F}_{2}\left(\psi _ { 2 } \left(\rho\left(\mathrm{y}_{\mathrm{n}}, \mathrm{y}_{\mathrm{n}}\right), \rho\left(\mathrm{y}_{\mathrm{n}}, \mathrm{y}_{\mathrm{n}+1}\right), \mathrm{d}\left(\mathrm{x}_{\mathrm{n}-1}, \mathrm{x}_{\mathrm{n}}\right)\right.\right. \text {, } \\
& \left.\left.\frac{\rho\left(\mathrm{y}_{\mathrm{n}}, \mathrm{y}_{\mathrm{n}}\right) \mathrm{d}\left(\mathrm{x}_{\mathrm{n}-1}, \mathrm{x}_{\mathrm{n}}\right)\left\{1+\rho\left(\mathrm{y}_{\mathrm{n}}, \mathrm{y}_{\mathrm{n}+1}\right)\right\}}{1+\rho\left(\mathrm{y}_{\mathrm{n}}, \mathrm{y}_{\mathrm{n}}\right)}\right)\right)
\end{aligned}
$$

or

$$
\begin{array}{r}
\rho_{n}^{m} \leq k \varphi_{2}\left(0, \rho_{\mathrm{n}}, \mathrm{d}_{\mathrm{n}-1}, 0\right)+\mathrm{F}_{2}\left(\psi_{2}\left(0, \rho_{\mathrm{n}}, \mathrm{d}_{\mathrm{n}-1}, 0\right)\right) \\
=k \varphi_{2}\left(0, \rho_{\mathrm{n}}, \mathrm{d}_{\mathrm{n}-1}, 0\right)
\end{array}
$$

For the coordinates of the point $\left(\rho_{\mathrm{n}}, \mathrm{d}_{\mathrm{n}-1}\right)$ we have $: \rho_{\mathrm{n}} \leq$ $\mathrm{d}_{\mathrm{n}-1}, \forall n \in N$, because ; in case that

$\rho_{\mathrm{n}}>\mathrm{d}_{\mathrm{n}-1}$ for some $\mathrm{n}$, if we replace the coordinates with $\rho_{\mathrm{n}}$ and apply the property (a) and (b) of $\varphi_{2}$

we get

$\rho_{n}^{m} \leq k \varphi_{2}\left(\rho_{\mathrm{n}}, \rho_{\mathrm{n}}, \rho_{\mathrm{n}}, \rho_{\mathrm{n}}\right) \leq \mathrm{k} \rho_{n}^{m}$

This is impossible since $0 \leq \mathrm{k}<1$.

Replacing on the right hand side of (3), the coor1dinates with $\rho_{\mathrm{n}-1}$ and applying properties (a) and (b) of $\varphi_{2}$ we get :

$\rho_{n}^{m} \leq k \varphi_{2}\left(\mathrm{~d}_{\mathrm{n}-1}, \mathrm{~d}_{\mathrm{n}-1}, \mathrm{~d}_{\mathrm{n}-1}, \mathrm{~d}_{\mathrm{n}-1}\right) \leq \mathrm{k} d_{n-1}^{m}$

Thus

$$
\rho_{\mathrm{n}} \leq \mathrm{m} \sqrt{\mathrm{k}} \mathrm{d}_{\mathrm{n}-1}
$$

By the inequality (1) for $\mathrm{x}=\mathrm{x}_{\mathrm{n}}$ and $\mathrm{y}=\mathrm{y}_{\mathrm{n}}$ we get

$\mathrm{d}^{\mathrm{m}}\left(\mathrm{x}_{\mathrm{n}}, \mathrm{x}_{\mathrm{n}+1}\right)=\mathrm{d}^{\mathrm{m}}\left(\mathrm{Sy}_{\mathrm{n}}, \mathrm{STx}_{\mathrm{n}}\right)$ 


$$
\begin{aligned}
& \leq \mathrm{k} \varphi_{1}\left(\mathrm{~d}\left(\mathrm{x}_{\mathrm{n}}, \mathrm{S} y_{\mathrm{n}}\right), \mathrm{d}\left(\mathrm{x}_{\mathrm{n}}, \mathrm{STx}_{\mathrm{n}}\right), \rho\left(y_{n}, T x_{n}\right),\right. \\
& \left.\frac{\mathrm{d}\left(\mathrm{x}_{\mathrm{n}}, \mathrm{Sy}_{\mathrm{n}}\right) \mathrm{d}\left(\mathrm{x}_{\mathrm{n}}, \mathrm{STx}_{\mathrm{n}}\right)\left\{1+\rho\left(y_{n}, T x_{n}\right)\right\}}{1+\mathrm{d}\left(\mathrm{x}_{\mathrm{n}}, \mathrm{Sy}_{\mathrm{n}}\right)}\right) \\
& +\mathrm{F}_{1}\left(\psi _ { 1 } \left(\mathrm{~d}\left(\mathrm{x}_{\mathrm{n}}, \mathrm{Sy}_{\mathrm{n}}\right), \mathrm{d}\left(\mathrm{x}_{\mathrm{n}}, \mathrm{STX}_{\mathrm{n}}\right), \rho\left(\mathrm{y}_{\mathrm{n}}, \mathrm{Tx}_{\mathrm{n}}\right),\right.\right. \\
& \left.\left.\frac{\mathrm{d}\left(\mathrm{x}_{\mathrm{n}}, \mathrm{Sy}_{\mathrm{n}}\right) \mathrm{d}\left(\mathrm{x}_{\mathrm{n}}, \mathrm{STx}_{\mathrm{n}}\right)\left\{1+\rho\left(y_{n}, T x_{n}\right)\right\}}{1+\mathrm{d}\left(\mathrm{x}_{\mathrm{n}}, \mathrm{Sy}_{\mathrm{n}}\right)}\right)\right) \\
& \leq \mathrm{k} \varphi_{1}\left(\mathrm{~d}\left(\mathrm{x}_{\mathrm{n}}, \mathrm{x}_{\mathrm{n}}\right), \mathrm{d}\left(\mathrm{x}_{\mathrm{n}}, \mathrm{x}_{\mathrm{n}+1}\right), \rho\left(\mathrm{y}_{\mathrm{n}}, \mathrm{y}_{\mathrm{n}+1}\right),\right. \\
& \left.\frac{d\left(x_{n}, x_{n}\right) d\left(x_{n}, x_{n+1}\right)\left\{1+\rho\left(y_{n}, y_{n+1}\right)\right\}}{1+d\left(x_{n}, x_{n}\right)}\right) \\
& +\mathrm{F}_{1}\left(\psi _ { 1 } \left(\mathrm{~d}\left(\mathrm{x}_{\mathrm{n}}, \mathrm{x}_{\mathrm{n}}\right), \mathrm{d}\left(\mathrm{x}_{\mathrm{n}}, \mathrm{x}_{\mathrm{n}+1}\right), \rho\left(\mathrm{y}_{\mathrm{n}}, \mathrm{y}_{\mathrm{n}+1}\right),\right.\right. \\
& \left.\frac{d\left(x_{n}, x_{n}\right) d\left(x_{n}, x_{n+1}\right)\left\{1+\rho\left(y_{n}, y_{n+1}\right)\right\}}{1+d\left(x_{n}, x_{n}\right)}\right)
\end{aligned}
$$

Or

$$
\begin{aligned}
d_{n}^{m} & \leq \mathrm{k} \varphi_{1}\left(0, \mathrm{~d}_{\mathrm{n}}, \rho_{\mathrm{n}}, 0\right)+\mathrm{F}_{1}\left(\psi_{1}\left(0, \mathrm{~d}_{\mathrm{n}}, \rho_{\mathrm{n}}, 0\right)\right) \\
& =\mathrm{k} \varphi_{1}\left(0, \mathrm{~d}_{\mathrm{n}}, \rho_{\mathrm{n}}, 0\right)
\end{aligned}
$$

In similar, we get :

$$
d_{n}^{m} \leq \mathrm{k} \varphi_{1}\left(\rho_{\mathrm{n}}, \rho_{\mathrm{n}}, \rho_{\mathrm{n}}, \rho_{\mathrm{n}}\right) \leq \mathrm{k} \rho_{n}^{m}
$$

Thus we will obtain :

$\mathrm{d}_{\mathrm{n}} \leq \mathrm{m} \sqrt{\mathrm{k}} \rho_{\mathrm{n}}$

By this inequality and (4) we get :

$$
\mathrm{d}_{\mathrm{n}} \leq \mathrm{m} \sqrt{\mathrm{k}}\left({ }^{\mathrm{m}} \sqrt{\mathrm{kd}_{\mathrm{n}-1}}\right) \leq \sqrt[m]{\mathrm{k}} \mathrm{d}_{\mathrm{n}-1}
$$

by the inequalities (4) and (5), using the mathematical induction, we obtain :

$$
\begin{aligned}
& \mathrm{d}\left(\mathrm{x}_{\mathrm{n}}, \mathrm{x}_{\mathrm{n}+1}\right)=\mathrm{d}_{\mathrm{n}} \leq \mathrm{q}^{\mathrm{n}-1} \mathrm{~d}_{\mathrm{i}} \\
& \rho\left(\mathrm{y}_{\mathrm{n}}, \mathrm{y}_{\mathrm{n}+1}\right)=\rho_{\mathrm{n}} \leq \mathrm{q}^{\mathrm{n}-1} \mathrm{~d}_{1}
\end{aligned}
$$

Where $\mathrm{q}=\mathrm{m}_{\sqrt{\mathrm{k}}}<1$

Thus the sequences $\left(\mathrm{x}_{\mathrm{n}}\right)$ and $\left(\mathrm{y}_{\mathrm{n}}\right)$ are Cauchy sequences , since the Metric space Let $(\mathrm{X}, \mathrm{d}),(\mathrm{Y}, \rho)$ complete metric spaces we have :

$$
\begin{aligned}
& \lim _{n \rightarrow \infty} x_{n}=\alpha \in X, \lim _{n \rightarrow \infty} y_{n}=\beta \in \mathrm{Y} \\
& \text { by }(1) \text { for } \mathrm{y}= \beta \text { and } \mathrm{x}=\mathrm{x}_{\mathrm{n}} \text { we get : } \\
& \mathrm{d}^{\mathrm{m}}\left(\mathrm{S} \beta, \mathrm{x}_{\mathrm{n}+1}\right) \leq \mathrm{k} \varphi_{1}\left(\mathrm{~d}\left(\mathrm{x}_{\mathrm{n}}, \mathrm{S} \beta\right), \mathrm{d}\left(\mathrm{x}_{\mathrm{n}}, \mathrm{x}_{\mathrm{n}+1}\right), \rho\left(\beta, \mathrm{y}_{\mathrm{n}+1}\right),\right. \\
&\left.\frac{\mathrm{d}\left(\mathrm{x}_{\mathrm{n}}, \mathrm{S} \beta\right) \mathrm{d}\left(\mathrm{x}_{\mathrm{n}}, \mathrm{x}_{\mathrm{n}+1}\right)\left\{1+\rho\left(\beta, \mathrm{y}_{\mathrm{n}+1}\right)\right\}}{1+\mathrm{d}\left(\mathrm{x}_{\mathrm{n}}, \mathrm{S} \beta\right)}\right) \\
&+\mathrm{F}_{1}\left(\psi _ { 1 } \left(\mathrm{~d}\left(\mathrm{x}_{\mathrm{n}}, \mathrm{S} \beta\right), \mathrm{d}\left(\mathrm{x}_{\mathrm{n}}, \mathrm{x}_{\mathrm{n}+1}\right), \rho\left(\beta, \mathrm{y}_{\mathrm{n}+1}\right),\right.\right. \\
&\left.\left.\frac{\mathrm{d}\left(\mathrm{x}_{\mathrm{n}}, \mathrm{S} \beta\right) \mathrm{d}\left(\mathrm{x}_{\mathrm{n}}, \mathrm{x}_{\mathrm{n}+1}\right)\left\{1+\rho\left(\beta, \mathrm{y}_{\mathrm{n}+1}\right)\right\}}{1+\mathrm{d}\left(\mathrm{x}_{\mathrm{n}}, \mathrm{S} \beta\right)}\right)\right)
\end{aligned}
$$

letting $\mathrm{n} \rightarrow \infty$, we get :

$$
\begin{aligned}
& \mathrm{d}^{\mathrm{m}}(\mathrm{S} \beta, \alpha) \leq \mathrm{k} \varphi_{1}(\mathrm{~d}(\alpha, \mathrm{S} \beta), 0,0,0)+\mathrm{F}(0) \\
& \leq \mathrm{k} \varphi_{1}(\mathrm{~d}(\alpha, \mathrm{S} \beta) 0,0,0) \leq \mathrm{kd}^{\mathrm{m}}(\alpha, \mathrm{S} \beta)
\end{aligned}
$$

Or

$$
\mathrm{d}^{\mathrm{m}}(\mathrm{S} \beta, \alpha)=0 \quad \Leftrightarrow \mathrm{S} \beta=\alpha
$$

$$
\begin{aligned}
& \rho^{\mathrm{m}}\left(\mathrm{y}_{\mathrm{n}+1}, \mathrm{TS} \beta\right) \leq k \varphi_{2}\left(\rho\left(\beta, \mathrm{Tx}_{\mathrm{n}}\right), \rho(\beta, \mathrm{TS} \beta), \mathrm{d}\left(\mathrm{x}_{\mathrm{n}}, \mathrm{S} \beta\right),\right. \\
& \left.\frac{\rho\left(\beta, \mathrm{Tx}_{\mathrm{n}}\right) \mathrm{d}\left(\mathrm{x}_{\mathrm{n}}, \mathrm{S} \beta\right)\{1+\rho(\beta, \mathrm{TS} \beta)\}}{1+\rho\left(\beta, T x_{n}\right)}\right) \\
& +\mathrm{F}_{2}\left(\psi _ { 2 } \left(\rho\left(\beta, \mathrm{Tx}_{\mathrm{n}}\right), \rho(\beta, \mathrm{TS} \beta), \mathrm{d}\left(\mathrm{x}_{\mathrm{n}}, \mathrm{S} \beta\right)\right.\right. \text {, } \\
& \left.\left.\frac{\rho\left(\beta, \mathrm{Tx}_{\mathrm{n}}\right) \mathrm{d}\left(\mathrm{x}_{\mathrm{n}}, \mathrm{S} \beta\right)\{1+\rho(\beta, \mathrm{TS} \beta)\}}{1+\rho\left(\beta, T x_{n}\right)}\right)\right) \\
& \rho^{\mathrm{m}}\left(\mathrm{y}_{\mathrm{n}+1}, \mathrm{TS} \beta\right) \leq k \varphi_{2}\left(\rho\left(\beta, \mathrm{y}_{\mathrm{n}+1}\right), \rho(\beta, \mathrm{TS} \beta), \mathrm{d}\left(\mathrm{x}_{\mathrm{n}}, \mathrm{S} \beta\right),\right. \\
& \left.\frac{\rho\left(\beta, y_{n+1}\right) d\left(x_{n}, S \beta\right)\{1+\rho(\beta, T S \beta)\}}{1+\rho\left(\beta, y_{n+1}\right)}\right) \\
& +\mathrm{F}_{2}\left[\psi _ { 2 } \left(\rho\left(\beta, \mathrm{y}_{\mathrm{n}+1}\right), \rho(\beta, \mathrm{TS} \beta), \mathrm{d}\left(\mathrm{x}_{\mathrm{n}}, \mathrm{S} \beta\right),\right.\right. \\
& \left.\left.\frac{\rho\left(\beta, \mathrm{y}_{\mathrm{n}+1}\right) \mathrm{d}\left(\mathrm{x}_{\mathrm{n}}, \mathrm{S} \beta\right)\{1+\rho(\beta, \mathrm{TS} \beta)\}}{1+\rho\left(\beta, y_{n+1}\right)}\right)\right)
\end{aligned}
$$

letting $\mathrm{n} \rightarrow \infty$, and using(6) we get :

$\rho^{\mathrm{m}}(\beta, \mathrm{TS} \beta) \leq k \varphi_{2}(0, \rho(\beta, \mathrm{TS} \beta), 0,0)+\mathrm{F}(0)$

or

$$
\rho^{\mathrm{m}}(\beta, \mathrm{TS} \beta) \leq k \rho^{\mathrm{m}}(\beta, \mathrm{TS} \beta) \quad \Leftrightarrow \mathrm{TS} \beta=\beta
$$

by (6) and (7) it follows :

$$
\begin{aligned}
& \mathrm{TS} \beta=\mathrm{T} \alpha=\beta \\
& \mathrm{ST} \alpha=\mathrm{S} \beta=\alpha
\end{aligned}
$$

Thus we proved that the points $\alpha, \beta$ are fixed points of ST and TS respectively .

\section{UNIQUENESS}

suppose that ST has a second distinct fixed point $\alpha^{\prime}$ in X . By (1) for $\mathrm{x}=\alpha^{\prime}$ and $\mathrm{y}=\mathrm{T} \alpha$ we get :

$\mathrm{d}^{\mathrm{m}}\left(\mathrm{ST} \alpha, \mathrm{ST} \alpha^{\prime}\right)$

$\leq \mathrm{k} \varphi_{1}\left(\mathrm{~d}\left(\alpha^{\prime}, \mathrm{ST} \alpha\right), \mathrm{d}\left(\alpha^{\prime}, \mathrm{ST} \alpha^{\prime}\right), \rho\left(\mathrm{T} \alpha, \mathrm{T} \alpha^{\prime}\right)\right.$, $\left.\frac{\mathrm{d}\left(\alpha^{\prime}, \mathrm{ST} \alpha\right) \mathrm{d}\left(\alpha^{\prime}, \mathrm{ST} \alpha^{\prime}\right)\left\{1+\rho\left(\mathrm{T} \alpha, \mathrm{T} \alpha^{\prime}\right)\right\}}{1+\mathrm{d}\left(\alpha^{\prime}, \mathrm{ST} \alpha\right)}\right)$

$+\mathrm{F}_{1}\left(\psi_{1}\left(\mathrm{~d}\left(\alpha^{\prime}, \mathrm{ST} \alpha\right), \mathrm{d}\left(\alpha^{\prime}, \mathrm{ST} \alpha^{\prime}\right), \rho\left(\mathrm{T} \alpha, \mathrm{T} \alpha^{\prime}\right)\right.\right.$, $\left.\left.\frac{\mathrm{d}\left(\alpha^{\prime}, \mathrm{ST} \alpha\right) \mathrm{d}\left(\alpha^{\prime}, \mathrm{ST} \alpha^{\prime}\right)\left\{1+\rho\left(\mathrm{T} \alpha, \mathrm{T} \alpha^{\prime}\right)\right\}}{1+\mathrm{d}\left(\alpha^{\prime}, \mathrm{ST} \alpha\right)}\right)\right)$

$\mathrm{d}^{\mathrm{m}}\left(\mathrm{ST} \alpha, \mathrm{ST} \alpha^{\prime}\right) \leq \mathrm{k} \varphi_{1}\left(\mathrm{~d}\left(\alpha^{\prime}, \alpha\right), 0, \rho\left(\mathrm{T} \alpha, \mathrm{T} \alpha^{\prime}\right), 0\right)$

Or $+\mathrm{F}_{1}\left(\psi_{1}\left(\mathrm{~d}\left(\alpha^{\prime}, \alpha\right), 0, \rho\left(\mathrm{T} \alpha, \mathrm{T} \alpha^{\prime}\right), 0\right)\right)$

$$
\begin{aligned}
\mathrm{d}^{\mathrm{m}}\left(\alpha, \alpha^{\prime}\right) \leq & \mathrm{k} \varphi_{1}\left(\mathrm{~d}\left(\alpha^{\prime}, \alpha\right), 0, \rho\left(\mathrm{T} \alpha, \mathrm{T} \alpha^{\prime}\right), 0\right) \\
& +\mathrm{F}_{1}(0) \\
\leq & \mathrm{k} \rho^{\mathrm{m}}\left(\mathrm{T} \alpha, \mathrm{T} \alpha^{\prime}\right)
\end{aligned}
$$

By (2) for $\mathrm{x}=\alpha^{\prime}$ and $\mathrm{y}=\mathrm{T} \alpha$ we obtain :

$$
\begin{aligned}
& \rho^{\mathrm{m}}\left(\mathrm{T} \alpha^{\prime}, \mathrm{T} \alpha\right) \leq k \varphi_{2}\left(\rho\left(\mathrm{T} \alpha, \mathrm{T} \alpha^{\prime}\right), \rho(\mathrm{T} \alpha, \mathrm{T} \alpha), \mathrm{d}\left(\alpha^{\prime}, \alpha\right),\right. \\
& \left.\frac{\rho\left(\mathrm{T} \alpha, \mathrm{T} \alpha^{\prime}\right) \mathrm{d}\left(\alpha^{\prime}, \alpha\right)\{1+\rho(\mathrm{T} \alpha, \mathrm{T} \alpha)\}}{1+\rho\left(\mathrm{T} \alpha, \mathrm{T} \alpha^{\prime}\right)}\right) \\
& +\mathrm{F}_{2}\left(\psi _ { 2 } \left(\rho\left(\mathrm{T} \alpha \mathrm{T} \alpha^{\prime}\right), \rho(\mathrm{T} \alpha, \mathrm{T} \alpha), \mathrm{d}\left(\alpha, \alpha^{\prime}\right)\right.\right. \text {, } \\
& \left.\left.\frac{\rho\left(\mathrm{T} \alpha, \mathrm{T} \alpha^{\prime}\right) \mathrm{d}\left(\alpha^{\prime}, \alpha\right)\{1+\rho(\mathrm{T} \alpha, \mathrm{T} \alpha)\}}{1+\rho\left(\mathrm{T} \alpha, \mathrm{T} \alpha^{\prime}\right)}\right)\right) \\
& \rho^{\mathrm{m}}\left(\mathrm{T} \alpha^{\prime}, \mathrm{T} \alpha\right) \leq k \varphi_{2}\left(\rho\left(\mathrm{T} \alpha, \mathrm{T} \alpha^{\prime}\right), 0, \mathrm{~d}\left(\alpha^{\prime}, \alpha\right),\right. \\
& \left.\frac{\rho\left(\mathrm{T} \alpha, \mathrm{T} \alpha^{\prime}\right) \mathrm{d}\left(\alpha^{\prime}, \alpha\right)\{1+0\}}{1+\rho\left(\mathrm{T} \alpha, \mathrm{T} \alpha^{\prime}\right)}\right) \\
& +\mathrm{F}_{2}\left(\psi _ { 2 } \left(\rho\left(\mathrm{T} \alpha, \mathrm{T} \alpha^{\prime}\right), 0, \mathrm{~d}\left(\alpha, \alpha^{\prime}\right)\right.\right. \text {, } \\
& \left.\left.\frac{\rho\left(\mathrm{T} \alpha, \mathrm{T} \alpha^{\prime}\right) \mathrm{d}\left(\alpha^{\prime}, \alpha\right)\{1+0\}}{1+\rho\left(\mathrm{T} \alpha, \mathrm{T} \alpha^{\prime}\right)}\right)\right)
\end{aligned}
$$


or

$$
\begin{gathered}
\rho^{\mathrm{m}}\left(\mathrm{T} \alpha^{\prime}, \mathrm{T} \alpha\right) \leq k \varphi_{2}\left(\rho\left(\mathrm{T} \alpha^{\prime}, \mathrm{T} \alpha\right), 0, \mathrm{~d}\left(\alpha, \alpha^{\prime}\right)\right. \\
\left.\frac{\rho\left(\mathrm{T} \alpha, \mathrm{T} \alpha^{\prime}\right) \mathrm{d}\left(\alpha^{\prime}, \alpha\right)}{1+\rho\left(\mathrm{T} \alpha, \mathrm{T} \alpha^{\prime}\right)}\right) \\
\quad+\mathrm{F}_{2}(0) \\
\leq \quad \mathrm{kd}^{\mathrm{m}}\left(\alpha, \alpha^{\prime}\right) \rho^{\mathrm{m}}\left(\mathrm{T} \alpha^{\prime}, \mathrm{T} \alpha\right) \\
\leq k \varphi_{2}\left(\rho\left(\mathrm{T} \alpha^{\prime}, \mathrm{T} \alpha\right), 0, \mathrm{~d}\left(\alpha, \alpha^{\prime}\right)\right. \\
\left.\frac{\rho\left(\mathrm{T} \alpha, \mathrm{T} \alpha^{\prime}\right) \mathrm{d}\left(\alpha^{\prime}, \alpha\right)}{1+\rho\left(\mathrm{T} \alpha, \mathrm{T} \alpha^{\prime}\right)}\right) \\
\leq \\
\mathrm{kd}^{\mathrm{m}}\left(\alpha, \alpha^{\prime}\right)
\end{gathered}
$$

By (8) and (9) we get :

$$
\mathrm{d}^{\mathrm{m}}\left(\alpha, \alpha^{\prime}\right) \leq \mathrm{k}^{2} \mathrm{~d}^{\mathrm{m}}\left(\alpha, \alpha^{\prime}\right)
$$

it follows

$\mathrm{d}\left(\alpha, \alpha^{\prime}\right)=$ 0Thus we have again $\alpha=\alpha^{\prime}$. in same way, it is proved the inequality of $\beta$

\section{Hence Proved}

\section{References}

[1] B.Fisher,Fixedpointontwometricspaces,GlasnikMathem.16( 36)(1981),333-337.

[2] S.Č.Nešić,Afixedpointtheoremintwometricspaces,Bull.Math .Soc.Sci.Math.Roumanie,Tome44(94)(2001)No.3,253-257.

[3] V.Popa,Fixedpointsontwocompletemetricspaces,Zb.Rad.Prir od.-Mat.Fak.Sez.Mat.21,1(1991),83-93. 